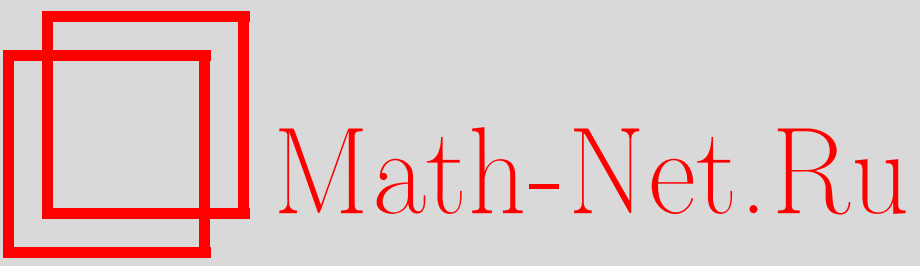

С. Ю. Оревков, Объем многогранника Ньютона дискриминанта, УМН, 1999, том 54, выпуск 5, 165-166

DOI: https://doi.org/10.4213/rm213

Использование Общероссийского математического портала Math-Net.Ru подразумевает, что вы прочитали и согласны с пользовательским соглашением

http://www.mathnet.ru/rus/agreement

Параметры загрузки:

IP : 54.174 .149 .18

26 апреля 2023 г., 12:47:03 


\title{
ОБЪЕМ МНОГОГРАННИКА НЬЮТОНА ДИСКРИМИНАНТА
}

\author{
С. Ю. ОревКов
}

1. Формулировка результата. Пусть $D_{n}=D_{n}\left(x_{0}, \ldots, x_{n}\right)$ - дискриминант, т.е. многочлен от $x_{0}, \ldots, x_{n}$, обращающийся в ноль тогда и только тогда, когда многочлен $\sum_{k=0}^{n} x_{k} t^{k}$ имеет кратный корень. Пример: $D_{2}(a, b, c)=b^{2}-4 a c$.

Многогранником Ньютона $\Delta(f)$ многочлена $f=\sum a_{u} x_{1}^{u_{1}} \cdots x_{N}^{u_{N}}$, где $u=\left(u_{1}, \ldots, u_{n}\right)$, называется выпуклая оболочка в $\mathbb{R}^{N}$ множества $\left\{u \in \mathbb{Z}^{N} \mid a_{u} \neq 0\right\}$. Если $V \in \mathbb{R}^{N}$ - аффинная $k$-мерная плоскость такая, что ранг решетки $V \cap \mathbb{Z}^{N}$ равен $k$, то $k$-мерный объем vol $k$ на плоскости $V$ будем нормировать так, чтобы объем фундаментального параллелепипеда решетки был равен единице.

Обозначим $\Delta\left(D_{n}\right)$ через $Q_{n}$. В силу очевидных однородности и квазиоднородности дискриминанта, $Q_{n}$ лежит в $(n-1)$-мерной плоскости

$$
u_{0}+\cdots+u_{n}=2(n-1), \quad u_{1}+2 u_{2}+\cdots+n u_{n}=n(n-1) .
$$

TEOPEMA 1. $\operatorname{vol}_{n-1} Q_{n}=2^{n-1} n^{n-2} / n$ ! .

TEOPEMA 2. $\operatorname{vol}_{n-2} \Delta\left(\bar{D}_{n}\right)=(n+6) 2^{n-3} n^{n-5} /(n-2) ! n p u n \geqslant 3$, где $\bar{D}_{n}\left(y_{0}, \ldots, y_{n-2}\right)$ - дискриминант многочлена $t^{n}+y_{n-2} t^{n-2}+\cdots+y_{0}$.

Пусть $A \subset \mathbb{Z}^{d}$ - множество из $n$ точек, $P_{A}$ - его выпуклая оболочка, $\operatorname{dim} P_{A}=d$. Следуя [1], обозначим через $\mathbf{C}^{A}$ пространство многочленов Лорана вида $\sum_{a \in A} x_{a} t^{a}$, где $t=\left(t_{1}, \ldots, t_{d}\right)$, $a=\left(a_{1}, \ldots, a_{d}\right), t^{a}=t_{1}^{a_{1}} \cdots t_{d}^{a_{d}}$, и определим дискриминант $D_{A}$ как многочлен от $n$ переменных $\left(x_{a}\right)_{a \in A}$ такой, что уравнение $D_{A}=0$ задает гиперповерхность в $\mathbf{C}^{A}$, являющуюся замыканием множества всех многочленов $f$, для которых гиперповерхность $\{f=0\}$ имеет особенность в торе $(\mathbf{C} \backslash 0)^{d}$. Соответственно, дискриминант $E_{A}$ задает замыкание множества многочленов, имеющих вырожденное ограничение хотя бы на одну из граней $P_{A}$ (подробности см. в [1]). Пусть $N=n-d-1=\operatorname{dim} \Delta\left(D_{A}\right)=\operatorname{dim} \Delta\left(E_{A}\right)$

TEOpema 3. $\operatorname{vol}_{N} \Delta\left(E_{A}\right)>\left(\prod_{k=1}^{d}(k+1)^{i_{k}}\right)(N-c) ! / N !$, əде $c=i_{0}-d-1 u i_{k}-$ чucлo точек из $A$, являющихся внутренними точками $k$-мерных граней многогранника $P_{A}$.

СлЕДСтвиЕ. Для любого $d$ существуют $C_{0}(d), C_{1}(d)>0$ maкие, что $\log \operatorname{vol} Q_{d, m} \geqslant$ $C_{0}(d)+C_{1}(d) m^{d}$, где $Q_{d, m}=\Delta\left(D_{A}\right)$ для $A=\left\{a \in \mathbb{Z}^{d} \mid a_{i} \geqslant 0, \sum a_{i} \leqslant m\right\}$.

Это дает отрицательный ответ на вопрос Е.И. Шустина о том, существуют ли константы $B_{0}(d), B_{1}(d)$ такие, что $\log \left(N ! \operatorname{vol} Q_{d, m}\right) \leqslant B_{0}(d)+B_{1}(d) m^{d}$, где $N=C_{n+d}^{d}-d-1=$ $\operatorname{dim} Q_{d, m}$. Положительный ответ давал бы ожидаемую верхнюю асимптотическую оценку числа жестких изотопических типов проективных вещественных гиперповерхностей степени $m$ при $m \rightarrow \infty$ (того же порядка, что и нижняя оценка, вытекающая из построений методом Виро).

2. Обозначения. Для $k \in \mathbb{Z}$ положим $\bar{k}=\{1, \ldots, k\}(\overline{0}=\varnothing)$. Через $S_{n}$ обозначим симметрическую группу: $S_{n}=\{\sigma: \bar{n} \rightarrow \bar{n} \mid \sigma(\bar{n})=\bar{n}\}$, через $\pi_{n}: \mathbb{R}^{n+1} \rightarrow \mathbb{R}^{n-1}$ - проекцию $\left(u_{0}, \ldots, u_{n}\right) \mapsto\left(u_{1}, \ldots, u_{n-1}\right)$. Для конечного множества $\alpha$ обозначим через \# $\alpha$ его мощность и положим $C_{\alpha}^{k}=\{\beta \subset \alpha \mid \# \beta=k\}$ (тогда \# $C_{\alpha}^{k}=C_{\# \alpha}^{k}-$ биномиальный коэффициент). Для $\alpha \subset \mathbb{Z}$ обозначим через $\mu_{\alpha}:\{1, \ldots, \# \alpha\} \rightarrow \alpha$ биекцию такую, что $\mu_{\alpha}(1)<\mu_{\alpha}(2)<\cdots$. Буква $m$ всегда будет обозначать $n-1$.

3. $Q_{n}$ как вторичный многогранник. Согласно результату Гельфанда-ЗелевинскогоКапранова [1], $Q_{n}$ комбинаторно эквивалентен $m$-кубу (напомним, что $m=n-1$ ), и его вершинами являются точки $\left\{q_{\alpha}\right\}_{\alpha} \subset \bar{m}$, где координаты $\left(q_{0}^{\alpha}, \ldots, q_{n}^{\alpha}\right)$ точки $q_{\alpha}$ определяются следующим образом. Если $\alpha=\left\{k_{1}, \ldots, k_{a}\right\}, 0=k_{0}<k_{1}<\cdots<k_{a}<k_{a+1}=n, k_{-1}=1$, $k_{a+2}=n-1$, то $q_{k}^{\alpha}=k_{i+1}-k_{i-1}$ при $k=k_{i} \in \alpha \cup\{0, n\}, q_{k}^{\alpha}=0$ при $k \notin \alpha \cup\{0, n\}$.

4. Триангуляция перекошенного куба. Пусть $p_{\alpha}=\left(p_{1}^{\alpha}, \ldots, p_{N}^{\alpha}\right)$ - точки в $\mathbb{R}^{N}$, индексированные подмножествами $\alpha \subset \bar{N}$, такие, что $p_{i}^{\alpha}>0$ при $i \in \alpha, p_{i}^{\alpha}=0$ при $i \notin \alpha$. Для $\sigma \in S_{N}$ обозначим через $s_{\sigma}$ симплекс, натянутьй на точки $p_{\sigma(\bar{k})}, k=0, \ldots, N$. 
Лемма 1. а) $\left\{s_{\sigma}\right\}_{\sigma \in S_{N}}-$ триангуляция некоторого (необязательно выпуклого) многогранника $P$, гомеоморфного кубу (значит, $\left.\operatorname{vol} P=\sum \operatorname{vol} s_{\sigma}\right)$.

б) Если выпуклая оболочка $P^{\prime}$ точек $p_{\alpha}$ комбинаторно әквивалентна кубу (т.е. для любого $i$ все точки $\left\{p_{\alpha}\right\}_{i \in \alpha}$ лежат на одной $(N-1)$-мерной грани многогранника $\left.P^{\prime}\right)$, mo $P^{\prime}=P$.

ДокАЗАТЕЛЬСтво. Проектируя из $p_{\bar{N}}$, зададим $P$ индуктивно как объединение конусов над пересечениями с координатными гиперплоскостями.

Пример: если $P$ - куб, то $s_{\sigma}=\left\{\left(x_{1}, \ldots, x_{N}\right) \in P \mid x_{\sigma(1)} \geqslant x_{\sigma(2)} \geqslant \cdots \geqslant x_{\sigma(N)}\right\}$.

5. Рекуррентное соотношение. Пусть $\left\{s_{\sigma}\right\}_{\sigma \in S_{m}}-$ триангуляция $Q_{n}$ из п. 4.

Лемма 2. $\operatorname{vol} \pi_{n}\left(s_{\sigma}\right)=\left(\prod_{k=1}^{n-1} q_{\sigma(k)}^{\sigma(\bar{k})}\right) /(n-1)$ ! .

ДокАЗАТЕЛЬСтво. $\pi_{n}\left(q_{\sigma(\overline{0})}\right)=\pi_{n}\left(q_{\varnothing}\right)=0$. Поэтому $m ! \operatorname{vol} \pi_{n}\left(s_{\sigma}\right)=\left|\operatorname{det} A_{\sigma}\right|$, где $A_{\sigma}$ - матрица, составленная из векторов $\left\{q_{\sigma(\bar{k})}\right\}_{k \in \bar{m}}$, записанных по столбцам. Остается заметить, что в $\sigma(k)$-й строке на $k$-м месте стоит $q_{\sigma(k)}^{\sigma(\bar{k})}$, а слева от него нули.

ЛЕмма 3. $v_{n}=n \sum_{k=1}^{n-1} C_{n-2}^{k-1} v_{k} v_{n-k}$, где $v_{1}=1, v_{n}=(n-1) ! \operatorname{vol} \pi_{n}\left(Q_{n}\right)$.

ДоказАтЕльство. Следует из лемм 1 и 2 , если $\sum_{\sigma \in S_{m}}$ представить в виде $\sum_{k \in \bar{m}} \sum_{\sigma \in S_{m}^{k}}$, где $S_{m}^{k}=\{\sigma \mid \sigma(1)=k\}$, а затем внутреннюю сумму заменить на трехкратную сумму, отвечающую биекции $C_{\bar{m} \backslash\{1\}}^{k-1} \times S_{k-1} \times S_{m-k} \rightarrow S_{m}^{k},\left(\alpha, \sigma_{1}, \sigma_{2}\right) \mapsto \sigma$, где $\sigma(1)=k, \sigma(i)=\mu_{\alpha}(\sigma(i))$ при $i<k, \sigma(i)=\mu \bar{m} \backslash(\alpha \cup\{k\})\left(\sigma_{2}(i-k)\right)$ при $i>k$.

6. Тождество. Биномиальное тождество Абеля можно записать в таком виде [2; п. 1.2.7]: $\alpha \beta \sum_{k=0}^{n} C_{n}^{k}(\alpha+k)^{k-1}(\beta+n-k)^{n-k-1}=(\alpha+\beta)(\alpha+\beta+n)^{n-1}$. Подставляя $\beta=-\alpha$, деля на $\alpha^{2}$ и переходя к пределу при $\alpha \rightarrow 0$, получаем

$$
\sum_{k=1}^{n-1} C_{n}^{k} k^{k-1}(n-k)^{n-k-1}=2(n-1) n^{n-2} .
$$

7. Доказательство теорем. Из (2) и леммы 3 индукцией получаем $v_{n}=2^{n-1} n^{n-2}$. Пусть $V$ - плоскость, заданная уравнениями (1). Разрешая (1) относительно $u_{0}, u_{1}$, получаем биекцию $j_{n}: \mathbb{Z}^{n-1} \rightarrow V \cap \mathbb{Z}^{n+1}$, причем $\left|\operatorname{det}\left(j_{n} \pi_{n}\right)\right|=n$, поэтому $n \operatorname{vol} Q_{n}=\operatorname{vol} \pi_{n}\left(Q_{n}\right)=$ $v_{n} /(n-1)$ !. Теорема 1 доказана. Теорема 2 доказывается аналогично: пользуясь рекуррентным соотношением $\bar{v}_{n}=n \sum_{k=2}^{n-1} C_{n-3}^{k-2} \bar{v}_{k} v_{n-k}$, находим $\bar{v}_{n}=(n+6) 2^{n-3} n^{n-4}$, где $\bar{v}_{n} /(n-2)$ ! - объем проекции $\Delta\left(\bar{D}_{n}\right)$ на плоскость $y_{n}=0$.

Теорема 3 вытекает из леммы $1 \mathrm{a})$. Согласно [1], $\Delta\left(E_{A}\right)$ является выпуклой оболочкой точек в $\mathbb{R}^{A}$, соответствующих триангуляциям многогранника $P_{A}$. Пусть $V$ - множество вершин $P_{A}$ $\left(i_{0}=\# V\right)$. Для каждого $\alpha \subset A \backslash V$ возьмем любую триангуляцию, для которой $\alpha \cup V$ является множеством вершин. Соответствуюшие им точки $\left\{q_{\alpha}\right\} \subset \mathbb{R}^{A}$ лежат на $M$-мерной плоскости $(M=N-c=\# A \backslash V)$ и удовлетворяют условиям леммы $1 \mathrm{a})$. Поэтому на них можно натянуть $M$ ! симплексов, объем каждого из которых $\geqslant \prod(k+1)^{i_{k}} / M$ ! (это следует из описания $Q_{A}$, приведенного в [1] и леммы 2). Точки $\left\{q_{\alpha}\right\}$ лежат на $M$-мерном сечении $Q_{A}\left(\operatorname{dim} Q_{A}=N\right)$, и это дает $M ! / N !$.

\section{СПИСОК ЛИТЕРАТУРЫ}

[1] Gelfand I. M., Kapranov M. M., Zelevinskii A. V. Discriminants, Resultants and Multidimensional Determinants. Boston: Birkhäuser, 1994. [2] Гульден Я., Джексон Д. Перечислительная комбинаторика. М.: Наука, 1990. 